\title{
Attitude of Medical Students and Doctors towards Complementary, Alternative and Integrative Medicine: A Single-Center, Questionnaire-Based Study
}

\author{
Anika Singh, Ashwin Kamath* \\ Department of Pharmacology, Kasturba Medical College, Mangalore, Manipal Academy of Higher Education, Manipal, Karnataka, India
}

Received January 12, 2021

Reviewed April 21, 2021

Accepted May 12, 2021
Objectives: Our study aimed to determine the attitudes of second- and final-year medical students and doctors (teaching faculty) of modern medicine towards complementary and alternative medicine (CAM) using the Complementary, Alternative, and Integrative Medicine Attitude Questionnaire (CAIMAQ).

Methods: We invited 248 second-year medical students, 245 final-year medical students, and 48 faculty members to participate in the study. The CAIMAQ consists of 30 items, divided into five categories assessing various aspects of CAM, and scored using a 7-point Likert scale. The median scores obtained were compared between groups; a p-value $<0.05$ was considered statistically significant.

Results: A total of 138 medical students and faculty responded and participated in the study, of which, 24 (17.4\%) were faculty, 40 (29\%) were final-year medical students and 74 (53.6\%) were second-year medical students. The overall attitude towards the various CAM concepts and therapies was positive. In general, the faculty were significantly less likely to consider referring patients for CAIM treatments, integrating them with conventional medicine, referring patients to alternative healthcare providers, considering the use of subtle energy fields as an ethical form of treatment, or considering CAIM treatments to be less invasive and harmful compared with conventional medicine. There was no significant difference in the attitudes of second- and final-year students.

Conclusion: The attitude of medical students and doctors towards CAM is positive, and although the medical faculty have reservations in recommending specific types of CAM therapies or integrating them with conventional care, building evidence for supporting CAM therapies in specific diseases is likely to increase its uptake among health care professionals.

Keywords: complementary medicine, attitude, students, doctors, knowledge

\section{INTRODUCTION}

Complementary and alternative medicine (CAM) systems are well known for their patient-centric approach to disease management, emphasizing not only on physical wellness but also the mental and social wellbeing. Although the basic tenets of any system of medicine are the same, the individualized as well as holistic approach of CAM has retained the confidence among the masses of its effectiveness [1-3]. The National Center for Complementary and Alternative Medicine classifies
CAM therapies into "alternative whole medical systems, mindbody interventions, biologically based therapies, manipulative and body-based methods, and energy therapies" [4]. As per a report by the World Health Organization (WHO), 75\% of the world's population still utilizes traditional systems of medicine [1]. The reliance on CAM is estimated to increase further with population growth, especially in a country like India, where utilization of CAM for daily medical needs is common practice. This continuing reliance on CAM has been attributed to lack of availability of essential medicines uniformly at all geographic 
locations, absence of health services by a qualified professional of conventional medicine, and poor socioeconomic status [5]. This is particularly true in patients suffering from chronic illness [6].

Given the widespread use of CAM and the gradual increase in the availability of evidence of its effectiveness in a number of disease conditions, one would expect that a preliminary knowledge regarding the CAM practices be imparted in medical schools teaching evidence-based modern allopathic medicine [7]. However, this has not been the case so far. The attitudes and opinions the students and practitioners of modern medicine build are, hence, likely to be based on their personal experiences, information from media, and views of their peers, teachers and mentors $[8,9]$. This has been probably responsible for the scepticism or uncertainty among medical students regarding the utility of CAM $[10,11]$. The concern also relates to lack of adequate knowledge regarding the possible interactions between drugs when a patient utilizes CAM along with modern medicines [12]. This fact is also supported by findings from physician surveys which show that majority of physicians would like to receive more education on CAM therapies [13, 14]. However, there is no adequate data regarding the hierarchical change in attitudes, from medical students to practitioners, towards CAM; such data would provide some meaningful information on the possible influence of the modern medical curriculum as well as teachers on the students' attitudes. In addition, national and international research findings regarding the usefulness and safety of various CAM modalities also influence the general opinion. The attitude of the medical students and doctors will determine to a large extent the uptake of such knowledge. An increased acceptance of CAM would suggest a need to ensure that basic information regarding CAM be provided in the medical curriculum so that informed decisions can be taken by the doctors.

Our study aims to determine the attitude of medical students and doctors towards CAM using the Complementary, Alternative, and Integrative Medicine Attitude Questionnaire (CAIMAQ) [15]. The objectives of our study are to assess the attitude of second- and final-year medical students and doctors (medical teaching faculty) towards CAM and determine the presence of any difference in attitudes among the studied groups. In India, the second-year medical students would have studied pre- (anatomy, physiology, and biochemistry) and paraclinical subjects (pathology, pharmacology, microbiology, etc.), with a brief exposure to the clinical subjects, by the end of the phase; the final-year students would have completed several rotations in all the major clinical subjects with a wider and indepth clinical exposure compared to the second-year students. The common educational environment shared by the study participants and the extent of their exposure to the theory and practice of medicine may indicate the change in attitudes as students progress to become practitioners.

\section{MATERIALS AND METHODS}

A cross-sectional questionnaire-based study was conducted at Kasturba Medical College, Mangalore, Karnataka, a medical institution providing multispecialty tertiary care in Southern India. The study participants consisted of all second-year and final-year medical students and faculty, who were willing to participate in the study. The study was initiated following approval from Kasturba Medical College Institutional Ethics Committee. After obtaining written informed consent, the study participants were instructed to complete the CAIMAQ questionnaire. The CAIMAQ, developed by Abbott et al. [15], consists of 30 items, grouped into five categories: "attitudes toward the desirability of CAIM therapies" (12 items); "attitudes toward progressive patient/physician health care roles" (7 items); "attitudes toward the mind-body-spirit connection" (4 items); "attitudes toward the principles of allostasis" (3 items); "attitudes toward a holistic understanding of disease" (4 items) [15]. The response to each item is recorded using a seven-point Likert scale, with one corresponding to strongly disagree and seven to strongly agree. No point is assigned to the response "don't know" and is treated as a missed response during analysis. The study proforma consisted of, besides the questionnaire, brief information regarding the study to be read by the participants, collection of data regarding age, gender, and whether second- or final-year medical student or faculty. Responses for all the completed items were included in the analysis.

\section{Statistical analysis}

The data from the questionnaire was entered into a Microsoft Office Excel file and then transferred to Statistical Package for Social Sciences (SPSS) file. SPSS Version 22.0 (IBM Corp, USA) was used for the analysis. Descriptive statistics such as frequencies, means and standard deviations for age, and median and interquartile range for test scores were used to summarize the data. The normality of data distribution was assessed using 
Shapiro-Wilk test. Since the data was not normally distributed ( $p<0.05)$, we used Kruskal-Wallis test for multiple group comparison. Mann-Whitney $U$ test was used to compare the results of two groups. A p-value less than 0.05 was considered statistically significant.

\section{RESULTS}

We invited 248 second-year medical students, 245 finalyear medical students, and 48 faculty members to participate in the study. A total of 138 (25.51\%) medical students and faculty responded and participated in the study, of which, 24 (17.4\%) were faculty, 40 (29\%) were final-year medical students and $74(53.6 \%)$ were second-year medical students. The mean age (standard deviation) of the students was 20.68 years (1.49) (minimum and maximum, 19 and 24 years, respectively). Of the 138 participants, $47 \%$ were females.

The attitudes of the second- and final-year medical students and faculty were compared for each of the CAIMAQ exploratory factors. A positive attitude towards the desirability of CAIM therapies was significantly lower among faculty compared with students (Table 1). In order to ascertain the presence of any gender difference in attitudes, we compared the responses of male and female student participants (Table 2). A positive attitude towards the desirability of CAIM therapies was significantly lower in females compared with male medical students. Also, the scores for attitudes toward a holistic understanding of the disease was significantly lower in females.

We then compared the responses of the study participants to each of the items in the CAIMAQ. Significant differences

Table 1. Attitudes of second- and final-year medical students and faculty towards complementary, alternative and integrative medicine determined using the CAIMAQ [15]

\begin{tabular}{|c|c|c|c|c|c|c|c|}
\hline \multirow[t]{2}{*}{ CAIMAQ factor } & \multicolumn{2}{|c|}{$\begin{array}{l}\text { Second year } \\
\qquad N=74\end{array}$} & \multicolumn{2}{|c|}{$\begin{array}{l}\text { Final year } \\
\mathrm{N}=40\end{array}$} & \multicolumn{2}{|c|}{$\begin{array}{l}\text { Faculty } \\
N=24\end{array}$} & \multirow[t]{2}{*}{ p-value* } \\
\hline & Median & IQR & Median & IQR & Median & IQR & \\
\hline $\begin{array}{l}\text { Attitudes toward progressive patient/ } \\
\text { physician health care roles }\end{array}$ & 5.9 & 5.1-6.1 & 5.3 & $4.9-6.0$ & 5.1 & 4.9-5.9 & 0.154 \\
\hline $\begin{array}{l}\text { Attitudes toward the principles of } \\
\text { allostasis }\end{array}$ & 5.2 & 4.3-5.7 & 5.0 & 4.3-5.7 & 5.3 & $5.0-5.5$ & 0.497 \\
\hline $\begin{array}{l}\text { Attitudes toward a holistic } \\
\text { understanding of disease }\end{array}$ & 5.0 & $4.0-5.5$ & 5.3 & $4.5-5.8$ & 5.0 & 4.9-5.3 & 0.263 \\
\hline
\end{tabular}

*Kruskal-Wallis test.

CAIMAQ, Complementary, Alternative and Integrative Medicine Attitude Questionnaire; IQR, Inter-Quartile Range.

Table 2. Attitudes of male and female second- and final-year medical students towards complementary, alternative and integrative medicine determined using the CAIMAQ [15]

\begin{tabular}{|c|c|c|c|c|c|}
\hline \multirow[t]{2}{*}{ CAIMAQ factor } & \multicolumn{2}{|c|}{$\begin{array}{l}\text { Male } \\
N=53\end{array}$} & \multicolumn{2}{|c|}{$\begin{array}{l}\text { Female } \\
\mathrm{N}=61\end{array}$} & \multirow[t]{2}{*}{ p-value* } \\
\hline & Median & IQR & Median & IQR & \\
\hline Attitudes toward the desirability of CAIM therapies & 5.0 & 4.4-5.7 & 4.7 & $3.8-5.2$ & 0.017 \\
\hline $\begin{array}{l}\text { Attitudes toward progressive patient/physician health care } \\
\text { roles }\end{array}$ & 5.7 & 5.1-6.1 & 5.7 & $5.0-6.1$ & 0.921 \\
\hline Attitudes toward the mind-body-spirit connection & 5.5 & $5.0-6.0$ & 5.5 & $5.0-6.0$ & 0.864 \\
\hline Attitudes toward the principles of allostasis & 5.3 & 4.3-6.0 & 5.0 & 4.3-5.7 & 0.464 \\
\hline Attitudes toward a holistic understanding of disease & 5.3 & 4.5-5.8 & 4.8 & 4.3-5.3 & 0.016 \\
\hline
\end{tabular}

*Mann-Whitney U test. 
in attitudes were seen for some of the items between the second- and final-year medical students and faculty (Table 3). In general, the faculty were significantly less likely to consider referring patients for CAIM treatments, integrating them with conventional medicine, referring patients to alternative health- care providers, considering use of subtle energy fields as an ethical form of treatment, or considering CAIM treatments to be less invasive and harmful compared with conventional medicine. Although statistically significant differences were seen for two items between the second- and final-year medical students,

Table 3. CAIMAQ item-wise differences in attitudes of second- and final-year medical students and faculty [15]

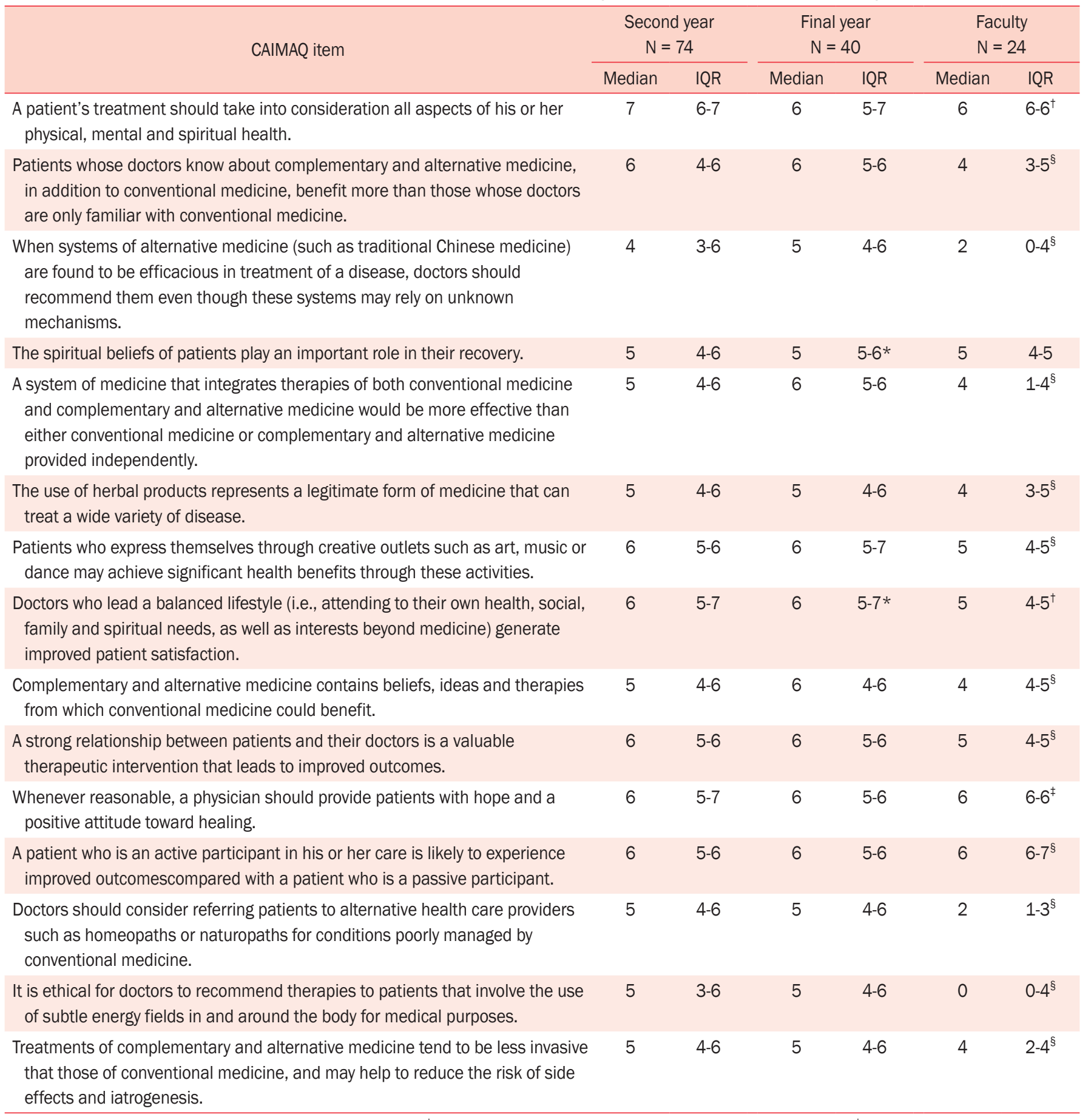

${ }^{*} p<0.05$ for second- and final-year student comparison; ${ }^{\dagger} p<0.05$ for second-year student and faculty comparison; ${ }^{\ddagger} p<0.05$ for final-year student and faculty comparison; ${ }^{\S} \mathrm{p}<0.05$ for second- and final-year student and faculty comparison. 
these differences are unlikely to be significant otherwise.

\section{DISCUSSION}

The findings of our study indicate that both medical students and faculty had a favorable attitude towards CAM, and in general, agreed to most of the statements regarding CAM concept and practice mentioned in the questionnaire. However, a significant difference in the attitudes was observed for statements involving the actual utilization or implementation of CAM techniques, with the faculty agreeing to these statements to a lesser extent compared with students. The three statements wherein the faculty expressed their disagreement (median score $<4)$ was use of alternative systems of medicine which are efficacious but their mechanism of action is unknown, referring of patients to CAM when the disease is poorly treated with conventional medicines, and the use of subtle energy fields as a treatment modality. A large component of this disagreement may be explained by the lack of availability of sufficient amount of evidence supporting specific CAM-based therapies and their effectiveness in a particular disease. Availability of published data supporting a treatment is crucial for its uptake into conventional practice; the fact that conventional modern medicine heavily relies on such data to build evidence, and there is still a large research gap for CAIM therapies, may explain this apparent negative attitude of actively practising medical faculty compared with those of medical students [16, 17]. Overall, the participants had a positive attitude towards the CAM concepts and considered these to be beneficial in patient management. The findings of our study are in agreement with that conducted among medical students in Saudi Arabia; the participants agreed to the views that use of CAM in combination with conventional medicine is likely to be useful when such combination treatment is indicated, and such use should be based on evidence of effectiveness [18].

The participating faculty believed that doctors should not recommend treatment modalities relying on unknown mechanisms even when such treatment is found to be efficacious for a particular disease. In contrast, a qualitative study among 19 general practitioners in England found that general practitioners favored the use of CAM in certain situations based on personal experience despite the lack of availability of adequate supporting evidence; moreover, the beneficial effects of these treatments were attributed to the placebo effect, which ultimately was positive in terms of patient outcome [18]. Although the findings seem different to the responses of faculty in our study, the lack of attribution of the beneficial effects of CAM to be due to an actual effect, rather than a placebo effect, suggests that the general practitioners do not consider these treatment modalities at par or as a suitable alternative to conventional medicines.

Our study found that students considered an integrated form of treatment involving both CAM and conventional medicine to be more beneficial than either individually. A recent study evaluated the effectiveness and safety of acupuncture for the treatment of Alzheimer's disease; the combination of acupuncture with drug therapy was found to be more beneficial effect in terms of general cognitive function and activities of daily living compared with drug therapy alone, although it may not be beneficial when used alone [19].

In the current study, students were more positively inclined in their view that a strong relationship between patients and their doctors would lead to improved outcomes, while the faculty were more likely to consider active participation by the patient in treatment to be beneficial; however, the general attitude towards both these aspects among the students and faculty was positive. This is consistent with a literature review done in 2018 which showed that trust and communication in the doctorpatient relationship are intricately linked to patient satisfaction and how they perceive the quality of the health services delivered [20].

A statistically significant difference in attitudes based on gender was found; male students had more positive attitudes towards the desirability of CAM therapies and the need for a holistic understanding of a disease. A similar study among students revealed no statistically significant gender-based differences, although women tended to have more positive attitudes than men [21]. However, the observed statistical differences are to be considered in the context of an overall positive attitude among both the genders.

There has been an increase in interest in CAM among the general population over the past decade; simultaneously, the need for evidence supporting the clinical effectiveness of the various CAM modalities is being increasingly recognized by medical professionals. Our study findings suggest that despite the widespread use of CAM, most general practitioners of conventional medicine are not confident in dealing with CAM due to lack of knowledge of CAM-related information and, therefore, did not agree with recommending such therapies. However, they do agree that CAM contains ideas and beliefs from 
which conventional medicine could benefit. More research would support further integration of CAM into conventional medicine as the benefit of these therapies is continually being identified. A positive attitude of medical students towards CAM and its related therapies suggests that provision of sufficient knowledge and introduction of CAM as a subject into the medical curriculum may influence CAM use and popularization among the to-be-future health professionals.

Our study has limitations. Although the participant population was formed by entry-level second-year medical students, final-year students about to obtain their degrees, and faculty who were currently practising medicine, all belonged to the same institution. Also, we considered faculty from different clinical specialities as a single cohort; however, the faculty views may be different based on the specific branch of medicine they practise. Response rates among final-year students and faculty were lower than that in second-year students. Some CAM modalities, such as massage therapy and subtle energy therapy, are less commonly practised and not well known; this might have influenced the participant responses. Some aspects of the questionnaire, such as the statement "The focus of a primary care physician should be on promoting health rather than treating disease" cannot be considered unique to CAM practice since this also forms a part of practice of modern medicine; hence, the positive responses to such statements may not necessarily be due to the attitude towards CAM, although, they do indicate points of convergence between the different systems of medicine. Future studies should also consider including practitioners of a specific CAM modality to determine how they perceive alternative CAM therapies.

\section{CONCLUSION}

Our study findings show that the overall attitude of medical students and faculty towards CAM is positive, with the former having a more positive attitude. Although the medical faculty have reservations in recommending specific types of CAM therapies or integrating them with conventional care, building evidence for supporting CAM therapies in specific diseases is likely to increase its uptake among health care professionals since their attitude towards the concepts and principles of CAM is positive. There is a need to consider providing preliminary education regarding CAM to students of conventional medicine.

\section{ACKNOWLEDGEMENT}

We thank the medical students and faculty for their active participation in the study.

\section{CONFLICT OF INTEREST}

The authors have no conflicts of interest to declare.

\section{FUNDING}

The study did not receive any funding from governmental or non-governmental sources.

\section{ORCID}

Anika Singh, https://orcid.org/0000-0001-5452-4967

Ashwin Kamath, https://orcid.org/0000-0002-9609-0199

\section{REFERENCES}

1. World Health Organization. WHO traditional medicine strategy: 2014-2023. Geneva: WHO; 2013. 76 p.

2. Adams J, Lui C, McLaughlin D. The use of complementary and alternative medicine in later life. Rev Clin Gerontol. 2009;19(4): 227-36.

3. Ernst E, Fugh-Berman A. Complementary and alternative medicine: what is it all about? Occup Environ Med. 2002;59(2):140-4; quiz 144, 84.

4. Ventola CL. Current issues regarding complementary and alternative medicine (CAM) in the United States: part 1: the widespread use of CAM and the need for better-informed health care professionals to provide patient counseling. P T. 2010;35(8):4618.

5. Pandey $M M$, Rastogi $S$, Rawat $A K$. Indian traditional ayurvedic system of medicine and nutritional supplementation. Evid Based Complement Alternat Med. 2013;2013:376327.

6. Shanmugasundaram S. Complementary and alternative therapies in palliative care: a transition from modern medicine to traditional medicine in India. J Cancer Pain Symptom Palliat. 2005; 1(4):25-9.

7. Torkelson C, Harris I, Kreitzer MJ. Evaluation of a complementary and alternative medicine rotation in medical school. Altern Ther Health Med. 2006;12(4):30-4.

8. Walker BF, Armson A, Hodgetts C, Jacques A, Chin FE, Kow G, et al. Knowledge, attitude, influences and use of complementary and alternative medicine (CAM) among chiropractic and nurs- 
ing students. Chiropr Man Therap. 2017;25:29.

9. Chez RA, Jonas WB, Crawford C. A survey of medical students' opinions about complementary and alternative medicine. Am J Obstet Gynecol. 2001;185(3):754-7.

10. Perkin MR, Pearcy RM, Fraser JS. A comparison of the attitudes shown by general practitioners, hospital doctors and medical students towards alternative medicine. J R Soc Med. 1994;87(9): 523-5.

11. Maha N, Shaw A. Academic doctors' views of complementary and alternative medicine (CAM) and its role within the NHS: an exploratory qualitative study. BMC Complement Altern Med. 2007;7:17.

12. Elmer GW, Lafferty WE, Tyree PT, Lind BK. Potential interactions between complementary/alternative products and conventional medicines in a Medicare population. Ann Pharmacother. 2007;41(10):1617-24.

13. Levine SM, Weber-Levine ML, Mayberry RM. Complementary and alternative medical practices: training, experience, and attitudes of a primary care medical school faculty. J Am Board Fam Pract. 2003;16(4):318-26.

14. Milden SP, Stokols D. Physicians' attitudes and practices regarding complementary and alternative medicine. Behav Med. 2004; 30(2):73-82.

15. Abbott RB, Hui KK, Hays RD, Mandel J, Goldstein M, Winegarden B, et al. Medical student attitudes toward complementary, alternative and integrative medicine. Evid Based Complement
Alternat Med. 2011;2011:985243.

16. Fischer FH, Lewith G, Witt CM, Linde K, von Ammon K, Cardini F, et al. High prevalence but limited evidence in complementary and alternative medicine: guidelines for future research. BMC Complement Altern Med. 2014;14:46.

17. Veziari Y, Leach MJ, Kumar S. Barriers to the conduct and application of research in complementary and alternative medicine: a systematic review. BMC Complement Altern Med. 2017;17(1): 166.

18. Alzahrani SH, Bashawri J, Salawati EM, Bakarman MA. Knowledge and attitudes towards complementary and alternative medicine among senior medical students in King Abdulaziz University, Saudi Arabia. Evid Based Complement Alternat Med. 2016;2016:9370721.

19. Jarvis A, Perry R, Smith D, Terry R, Peters S. General practitioners' beliefs about the clinical utility of complementary and alternative medicine. Prim Health Care Res Dev. 2015;16(3):24653.

20. Chandra S, Mohammadnezhad M, Ward P. Trust and communication in a doctor-patient relationship: a literature review. J Healthc Commun. 2018;3(3):36.

21. Chaterji R, Tractenberg RE, Amri H, Lumpkin M, Amorosi SB, Haramati A. A large-sample survey of first- and second-year medical student attitudes toward complementary and alternative medicine in the curriculum and in practice. Altern Ther Health Med. 2007;13(1):30-5. 